\title{
Predictors of high on-aspirin platelet reactivity in elderly patients with coronary artery disease
}

\author{
This article was published in the following Dove Press journal: \\ Clinical Interventions in Aging \\ 10 August 2017 \\ Number of times this article has been viewed
}

\author{
JW Zhang' \\ WW Liu' \\ Timothy A McCaffrey ${ }^{2}$ \\ $X Q \mathrm{He}^{\prime}$ \\ WY Liang' \\ XH Chen' \\ XR Feng' \\ Sidney W Fu \\ ML Liu' \\ 'Department of Geriatrics, Peking \\ University First Hospital, Beijing, \\ China; ${ }^{2}$ Department of Medicine, \\ George Washington University \\ Medical Center, Washington, DC, USA
}

Correspondence: ML Liu

Department of Geriatrics, Peking University First Hospital, No 8, Xishiku

Street, Xicheng, 100034, Beijing, China

Tel +86 I0 83572022

Fax +861066552095

Email liumeilin@hotmail.com
Objectives: Previous studies have illustrated the link between high on-aspirin platelet reactivity (HAPR) with increasing thrombotic risks. The aim of our study was to investigate relative risk factors of HAPR in elderly patients with coronary artery disease.

Methods: Elderly, hospitalized coronary artery disease patients on regular aspirin treatment were enrolled from January 2014 to September 2016. Medical records of each patient were collected, including demographic information, cardiovascular risk factors, concomitant drugs and routine biological parameters. Arachidonic acid (AA, $0.5 \mathrm{mg} / \mathrm{mL}$ ) and adenosine diphosphate (ADP, $5 \mu \mathrm{mol} / \mathrm{L}$ ) induced platelet aggregation were measured via light transmission assay (LTA) to evaluate antiplatelet responses, referred as LTA-AA and LTA-ADP.

Results: A total of 275 elderly patients were included, with mean age of $77.2 \pm 8.1$ years, and males accounted for $81.8 \%$. HAPR was defined as LTA-AA in the upper quartile of the enrolled population. HAPR patients tended to have lower renal function $(P=0.052)$. Higher serum uric acid (SUA) level, as well as lower platelet count, hemoglobin and hematocrit were observed in HAPR patients, with a higher proportion of diuretics use $(P<0.05)$. Multivariate analysis revealed that SUA (OR: 1.004, 95\% CI: $1.000-1.007, P=0.048)$, platelet count (OR: 0.994 , 95\% CI: $0.989-1.000, P=0.045$ ), hematocrit (OR: 0.921, 95\% CI: $0.864-0.981, P=0.011$ ) and concomitant P2Y12 receptor inhibitors use (OR: 1.965, 95\% CI: $1.075-3.592, P=0.028$ ) were correlated with HAPR. Spearman's correlation analysis demonstrated an inverse association of LTA-AA with hematocrit $(r=-0.234, P<0.001)$, hemoglobin $(r=-0.209, P<0.001)$ and estimated glomerular filtration rate $(r=-0.132, P=0.031)$.

Conclusion: SUA, platelet count, hematocrit and P2Y12 receptor inhibitors use were independently correlated with HAPR. These parameters might provide novel therapeutic targets for optimizing antiplatelet therapy.

Keywords: aspirin, platelet reactivity, elderly, risk factors,coronary artery disease

\section{Introduction}

Thrombosis, considered as pathological hemostasis, is one of the severe complications for coronary artery disease (CAD), which poses a great threat to public health. Platelet activation plays crucial roles in the process of thrombosis. For one thing, during the early stage of thrombosis, vascular injury initiates the recruitment of platelets to the damaged sites, then the activated platelets adhere to the vessel wall and release platelet agonists, which promote additional platelet activation and aggregation. For another, platelets exert procoagulant effects and release mediators that support the recruitment of leukocytes. ${ }^{1,2}$ In addition, recent studies revealed that platelet-derived mRNAs, miRNAs and exosomes are also involved in thrombus formation. ${ }^{3}$

Aspirin, exerting inhibitory effects on platelet activation by acetylating the serine 529 residue of cyclooxygenase $(\mathrm{COX})$, reduces the risk of myocardial infarction 
and stroke, and is thus widely used in the prevention and treatment of cardiovascular diseases. ${ }^{4,5}$ However, individual variations in the platelet response to aspirin limits its use in some patients, including patients who experience ischemic events despite regular aspirin therapy. ${ }^{6-8}$

High on-aspirin platelet reactivity (HAPR) is defined as insufficient inhibition of platelet activation in patients on regular aspirin treatment. As a consequence, aspirin fails to prevent thrombotic complications in a significant proportion of patients, in the range of $7 \%-20 \%$ of adults. ${ }^{9,10}$ Several mechanisms of HAPR have been put forward, such as the decreased biological availability, drug-drug interactions, inadequate doses or poor compliance, reduced absorption due to advanced age or concurrent proton pump inhibitors (PPIs), non-platelet thromboxane A2 (TXA2) synthesis, accelerated platelet turnover, polymorphism of $\mathrm{COX} /$ glycoprotein II $\beta /$ III $\alpha(\mathrm{GPII} / \mathrm{III} \alpha) / \mathrm{P} 2 \mathrm{Y} 12$ receptors/collagen receptors and others. ${ }^{7,11,12}$

The link between HAPR with increasing risk of ischemic events has been demonstrated in previous studies. ${ }^{13,14}$ A number of platelet function tests (PFTs) have been developed to distinguish HAPR patients, intending to reduce thrombotic risks as well as to guide individual treatment, ${ }^{15,16}$ among which light transmission assay (LTA) was regarded as the traditional "gold standard" of PFTs. Using different platelet agonists, such as arachidonic acid (AA), adenosine diphosphate (ADP), collagen and epinephrine, platelet inhibition due to aspirin or thienopyridines could be distinguished. However, controversies still existed in optimal cut-off for aspirin response, which should be capable of predicting both thrombotic events and bleeding complications. ${ }^{17}$

Older age was associated with an increasing incidence of high platelet reactivity (HPR) in patients receiving antiplatelet therapy, which might partially elucidate the relatively higher thrombosis risks and poorer prognosis in this particular population. ${ }^{18,19}$ In this study, we aimed to investigate potential risk factors for HAPR in elderly CAD patients (aged >60 years), thus identifying "at-risk" patients and promoting the optimization of antiplatelet therapy.

\section{Methods and materials Study design}

Elderly patients on regular aspirin treatment $(100 \mathrm{mg} / \mathrm{d})$, hospitalized in the Department of Geriatrics of Peking University First Hospital were enrolled from January 2014 to September 2016. Inclusion criteria were as follows:

1. Age: $>60$ years.
2. Presence of at least one of the following: stable angina pectoris; acute coronary syndrome (ACS); previous percutaneous coronary intervention (PCI); coronary artery bypass graft; confirmed coronary atherosclerotic plaques via computed tomography angiography or coronary angiography.

3. Platelet count in the range between $75 \times 10^{9} / \mathrm{L}$ and $600 \times 10^{9} / \mathrm{L}$.

Patients were excluded when there existed contraindications for aspirin, concomitant with non-steroidal anti-inflammatory drugs, GPII $\beta / \mathrm{III} \alpha$ receptor inhibitors, Vitamin K antagonists or novel anticoagulants; patients with severe renal or liver dysfunction, peptic ulcer or history of gastrointestinal hemorrhage were excluded.

This study complied with the Declaration of Helsinki, and the protocol was approved by the Ethical Review Committee of Peking University First Hospital. Written informed consent was obtained from all the enrolled participants.

\section{Light transmission assay}

All patients were on aspirin $100 \mathrm{mg}$ /day treatment for at least 1 week before evaluating platelet function by LTA. LTA using AA, $0.5 \mathrm{mg} / \mathrm{mL}$ induced platelet aggregation (LTA-AA) was measured in order to evaluate aspirin responses as described. ${ }^{20,21}$ Peripheral blood samples were drawn in a sodium citrate tube (1:9), and all measurements were conducted within 2 hours. Platelet-rich plasma (PRP) and platelet-poor plasma (PPP), used as reference, were prepared through centrifugation at separate speeds (200× g $10 \mathrm{~min}$ for PRP; 2,000× g $10 \mathrm{~min}$ for PPP). AAinduced platelet aggregation was performed using an LBY-NJ4 platelet aggregometer (PRECIL, Beijing, China). The percentage of platelet aggregation was defined as the maximal light transmittance after AA addition. Besides this, ADP, $5 \mu \mathrm{mol} / \mathrm{L}$ induced platelet aggregation (LTA-ADP) was also measured to exclude patients with poor response to thienopyridines. ${ }^{22,23}$ Platelet aggregation results were further normalized based on platelet counts of each blood sample to mitigate bias.

\section{Hospitalization records collection}

For each patient, detailed medical records during the hospitalization were obtained from the electronic system, including age, gender, cardiovascular disease status, cardiovascular risk factors, combined drugs, co-morbidities, routine biological parameters such as routine blood test, glucose, coagulation function, renal or liver function parameters, uric acid, lipids and others (Table 1). 
Table I Clinical features in patients with different platelet reactivity status

\begin{tabular}{|c|c|c|c|c|}
\hline Clinical features & LAPR ( $n=69)$ & MAPR $(n=138)$ & HAPR $(n=68)$ & $P$-value \\
\hline Age (years) & $76.0 \pm 8.6$ & $77.2 \pm 8.3$ & $78.4 \pm 7.2$ & 0.266 \\
\hline Female gender, $\mathrm{n}(\%)$ & $17(24.6)$ & $24(17.4)$ & $9(13.2)$ & 0.217 \\
\hline BMI $\left(\mathrm{kg} / \mathrm{m}^{2}\right)$ & $24.9 \pm 3.7$ & $24.5 \pm 3.2$ & $24.5 \pm 3.0$ & 0.596 \\
\hline Previous PCl, n (\%) & $38(55.1)$ & $80(58.0)$ & $38(55.9)$ & 0.912 \\
\hline Hypertension, n (\%) & 51 (73.9) & $102(73.9)$ & $53(38.4)$ & 0.798 \\
\hline Diabetes, n (\%) & $28(40.6)$ & $59(42.8)$ & $33(48.5)$ & 0.617 \\
\hline Ischemic cerebrovascular disease, $\mathrm{n}(\%)$ & $14(20.3)$ & $42(30.4)$ & $24(35.3)$ & 0.137 \\
\hline Hyperlipidemia, n (\%) & $57(82.6)$ & $115(83.3)$ & $59(86.8)$ & 0.767 \\
\hline Peripheral artery disease, $\mathrm{n}(\%)$ & $57(82.6)$ & III (80.4) & $54(79.4)$ & 0.887 \\
\hline Current smoking, n (\%) & $13(18.8)$ & $16(11.6)$ & $9(13.2)$ & 0.358 \\
\hline LVEF (\%) & $60.0(32.0-70.0)$ & $61.0(40.0-80.0)$ & $61.0(43.0-77.0)$ & 0.861 \\
\hline eGFR (mL/min) & $66.2 \pm 16.9$ & $65.0 \pm 15.2$ & $59.8 \pm 18.2$ & 0.052 \\
\hline 25-(OH)-D (pg/mL) & $40.5 \pm 17.0$ & $40.6 \pm 16.2$ & $43.4 \pm 16.6$ & 0.410 \\
\hline HbAlc (\%) & $6.0(5.4-9.0)$ & $6.0(5.2-9.8)$ & $6.1(5.2-8.4)$ & 0.478 \\
\hline Hcy $(\mu \mathrm{mol} / \mathrm{L})$ & $14.7 \pm 5.7$ & $|4.2 \pm 6|$. & $13.2 \pm 4.3$ & 0.185 \\
\hline hsCRP (mg/L) & $1.9(0.0-16.1)$ & $2.8(0.0-31.5)$ & $2.5(0.0-19.7)$ & 0.951 \\
\hline $\mathrm{TG}(\mathrm{mmol} / \mathrm{L})$ & $1.2(0.4-3.5)$ & I.4 (0.4-5.6) & $1.2(0.4-4.8)$ & 0.253 \\
\hline $\mathrm{TCHO}(\mathrm{mmol} / \mathrm{L})$ & $3.4(2.4-5.1)$ & $3.4(1.7-6.4)$ & $3.4(2.0-5.9)$ & 0.735 \\
\hline HDL-C (mmol/L) & $1.0(0.7-1.7)$ & $1.0(0.4-4.8)$ & $1.0(0.5-2.1)$ & 0.243 \\
\hline LDL-C (mmol/L) & $1.9(0.5-3.0)$ & $1.8(0.9-5.2)$ & $1.9(1.1-4.3)$ & 0.418 \\
\hline SUA $(\mu \mathrm{mol} / \mathrm{L})$ & $344.2 \pm 69.5$ & $337.9 \pm 85.2$ & $368.0 \pm 89.7$ & $0.010 *$ \\
\hline $\mathrm{PLT} \times 10^{9} / \mathrm{L}$ & $202.9 \pm 93.6$ & $186.6 \pm 53.2$ & $|72.4 \pm 5| .2$ & $0.025^{*}$ \\
\hline MPV (fl) & $8.5(6.9-13.8)$ & $8.4(6.2-12.4)$ & $8.6(7.0-11.3)$ & 0.651 \\
\hline $\mathrm{Hb}(\mathrm{g} / \mathrm{L})$ & $134.6 \pm 16.6$ & $132.1 \pm|6|$. & $126.7 \pm 17.4$ & $0.033^{*}$ \\
\hline $\mathrm{Hct}(\%)$ & $39.0 \pm 4.6$ & $38.1 \pm 4.6$ & $36.4 \pm 5.0$ & $0.005^{* *}$ \\
\hline PT (s) & $10.9 \pm 1.5$ & $11.2 \pm 2.9$ & $11.5 \pm 3.0$ & 0.981 \\
\hline APTT (s) & $32.7 \pm 5.6$ & $31.9 \pm 4.9$ & $32.3 \pm 4.7$ & 0.804 \\
\hline FIB-c (g/L) & $2.9(1.8-5.5)$ & $2.8(1.7-4.4)$ & $2.9(1.3-6.1)$ & 0.552 \\
\hline FDP $(\mathrm{mg} / \mathrm{L})$ & $1.3(0.0-7.4)$ & $1.5(0.0-31.4)$ & I.8 (0.0-1।.3) & 0.112 \\
\hline Thienopyridines, $\mathrm{n}(\%)$ & $29(42.0)$ & $69(50.0)$ & $4 I(60.3)$ & 0.099 \\
\hline Statins, n (\%) & $64(92.7)$ & $130(94.2)$ & $65(95.6)$ & 0.778 \\
\hline ACEI/ARB, n (\%) & $29(18.8)$ & $60(20.3)$ & $35(20.6)$ & 0.442 \\
\hline$\beta$-blockers, n (\%) & $50(72.4)$ & $94(68.1)$ & $49(72.1)$ & 0.753 \\
\hline CCB, n (\%) & $27(39.1)$ & $53(38.4)$ & $29(42.6)$ & 0.839 \\
\hline Diuretics, n (\%) & $8(11.6)$ & $18(13.0)$ & $17(25.0)$ & $0.048^{*}$ \\
\hline Nitrates, n (\%) & $25(36.2)$ & $55(39.9)$ & $26(38.2)$ & 0.878 \\
\hline Hypoglycemics, n (\%) & $19(27.5)$ & 44 (31.9) & $23(33.8)$ & 0.710 \\
\hline PPI, n (\%) & $21(30.4)$ & $43(31.2)$ & $24(35.3)$ & 0.796 \\
\hline
\end{tabular}

Notes: Values are mean \pm SD or median (range) unless stated otherwise. $* P<0.05$, $* * P<0.01$.

Abbreviations: ACEl, angiotensin-converting enzyme inhibitor; APTT, activated prothromboplastin time; ARB, angiotensin receptor antagonist; BMI, body mass index; CCB, calcium channel blockers; eGFR, estimated glomerular filtration rate; FDP, fibrin degradation product; Fib-c, Fibrinogen c; HAPR, high on-aspirin platelet reactivity; $\mathrm{Hb}$, hemoglobin; HbAlc, Hemoglobin Alc; Hct, hematocrit; Hcy, homocysteine; HDL-C, high density lipoprotein cholesterol; hsCRP, hypersensitive C reactive protein; LAPR, low on-aspirin platelet reactivity; LDL-C, low density lipoprotein cholesterol; LVEF, left ventricular ejection fraction; MAPR, moderate on-aspirin platelet reactivity; MPV, mean platelet volume; PCl, percutaneous coronary intervention; PLT, platelet count; PPI, proton pump inhibitors; PT, prothrombin time; SUA, serum uric acid; TCHO, total cholesterol; TG, triglycerides.

\section{Statistical analysis}

Continuous variables were expressed as mean \pm SD or median (range), while categorical variables were described as frequency and percentage. Conformity to normal distribution was evaluated for continuous variables using both Kolmogorov-Smirnov and Shapiro-Wilk tests. One-way analysis of variance test or nonparametric Kruskal-Wallis test was used to make comparisons for continuous variables, while chi-square or Fisher exact test was applied for categorical variables. Chi-square and linear trend test was performed to make comparison for age composition in patients with different platelet reactivity status. Receiver operating characteristic (ROC) curve and multivariate logistic regression analysis was performed to investigate risk factors for HAPR, and Spearman's correlation test was used to identify the factors correlated with LTA-AA. A 2-tailed $P$-value $<0.05$ was considered as statistically significant for all analysis executed. Statistical analysis was carried out using SPSS version 14.0 software (SPSS Inc., Chicago, IL, USA). 


\section{Results}

\section{Enrollment}

A total of 289 elderly CAD patients on regular aspirin treatment were enrolled, but 14 patients with high onclopidogrel platelet reactivity $(5 \mu \mathrm{mol} / \mathrm{L}$ ADP-induced platelet aggregation more than 50\%) were excluded to avoid bias, resulting in a total of 275 elderly CAD patients with the mean age of $77.2 \pm 8.1$ years. Males accounted for $81.8 \%$ (225 of 275), and 156 of the 275 CAD patients $(56.7 \%)$ had received PCI previously. The enrollment flowchart is shown in Figure S1.

\section{Distribution of platelet aggregation}

Light transmission assay of AA-induced platelet aggregation (LTA-AA) was measured to evaluate aspirin response, with the quartiles $9.54 \%, 11.63 \%$ and $13.93 \%$ of residual platelet aggregation respectively. HAPR was defined as LTA-AA in the upper quartile, that is, more than $13.93 \%$ residual platelet aggregation $(n=68)$. Meanwhile, low onaspirin platelet reactivity (LAPR) was defined as LTA-AA in the lower quartile of the enrolled population $(\leq 9.54 \%$, $\mathrm{n}=69$ ). The remaining 138 patients in the middle 2 quartiles were assigned into moderate on-aspirin platelet reactivity (MAPR) group.

As shown in Figure 1A, age composition varied among LAPR, MAPR and HAPR patients, and the proportion of senile patients (aged $\geq 80$ years) increased gradually, from LAPR group to HAPR group ( $P$ for linear trend $=0.046$ ). Distribution of LTA-AA and LTA-ADP in patients receiving dual antiplatelet therapy (DAPT) is shown in Figure 1B.

\section{Clinical features of HAPR}

Patients were divided into 3 groups, HAPR, MAPR and LAPR, according to quartiles of LTA-AA. Comparisons were made in terms of age, gender composition, cardiovascular risk factors, co-morbidities, routine biological parameters and concomitant drugs.

As shown in Table 1, HAPR patients had a tendency toward reduced renal function $(P=0.052)$. Higher serum uric acid (SUA), as well as lower platelet count, hematocrit and hemoglobin were observed in HAPR patients $(P<0.05)$. In terms of combined drugs, diuretics use was more frequently prescribed in HAPR patients $(P<0.05)$.

The cut-off for hyperuricemia in the elderly was defined as the SUA concentration $>7 \mathrm{mg} / \mathrm{dL}(416.5 \mathrm{mmol} / \mathrm{L}){ }^{24}$ Patients who met that criteria $(n=47)$ for hyperuricemia were more common in HAPR patients, when compared with nonHAPR patients $(14.5 \%, 12.3 \%, 29.4 \%$ for LAPR, MAPR and HAPR, $P=0.023)$.

\section{Multivariate regression analysis}

Predictive value for HAPR of each of the variables listed in Table 1 with a $P$-value $<0.10$ were evaluated using ROC curve, including SUA, platelet count, estimated glomerular filtration rate (eGFR), hemoglobin and hematocrit. Areas under the ROC curve are exhibited in Table S1. Furthermore, a combination of these variables had an improved predictive value when compared with each variable alone (area under the curve [AUC]: $0.661,95 \% \mathrm{CI}: 0.581-0.740, P<0.001)$.

To investigate related factors for HAPR, multivariate regression analysis was performed. Previously reported

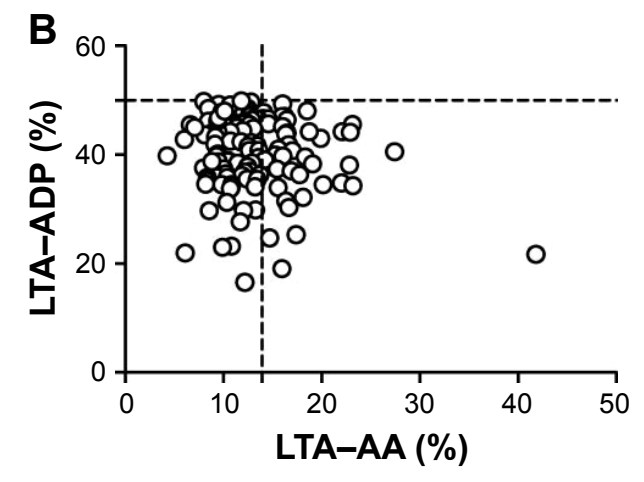

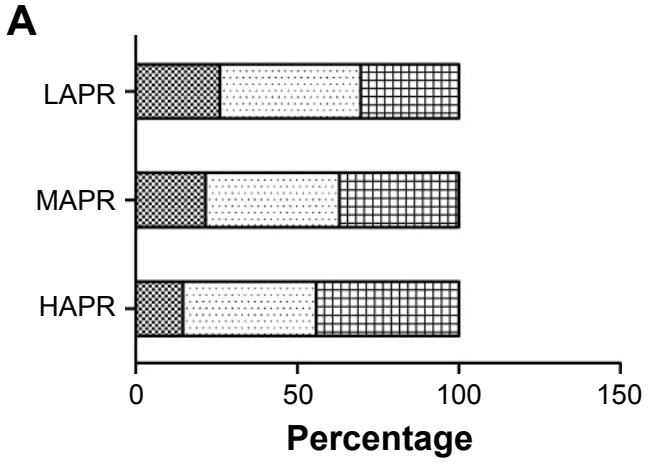

ㅍ... Aged $60-69$ years
… Aged $70-79$ years
ㅍ Aged $\geq 80$ years

Figure I Light transmission assay.

Notes: (A) Patients were divided into 3 groups according to the quartile of LTA-AA, and age composition varied among LAPR, MAPR and HAPR patients. The percentage of senile patients (aged more than or equal to 80 years) increased gradually, from LAPR group to HAPR group ( $P$ for linear trend $=0.046)$. (B) The distribution of LTA-AA and LTA-ADP in patients receiving dual antiplatelet therapy. The vertical dotted line indicates the cut-off of HAPR (I3.93\%), while the horizontal line marked the cut-off for poor clopidogrel response (50\%). HAPR (LTA-AA >13.93\%, $n=68)$; LAPR (LTA-AA $\leq 9.54 \%, n=69)$; MAPR $(9.54 \%<$ LTA-AA $\leq 13.93 \%, n=138)$.

Abbreviations: ADP, adenosine diphosphate; HAPR, high on-aspirin platelet reactivity; LTA-AA, light transmission assay-arachidonic acid; LAPR, low on-aspirin platelet reactivity; MAPR, moderate on-aspirin platelet reactivity. 
Table 2 Risk factors associated with HAPR

\begin{tabular}{|c|c|c|c|c|c|c|c|}
\hline Variables & B & SE & Wald & $P$-value & OR & 95\% Lower & 95\% Upper \\
\hline SUA & 0.00 & 0.002 & 3.920 & $0.048 *$ & $\mathrm{I} .004$ & 1.000 & 1.007 \\
\hline PLT & -0.01 & 0.003 & 4.011 & $0.045^{*}$ & 0.994 & 0.989 & 1.000 \\
\hline $\mathrm{Hct}$ & -0.08 & 0.032 & 6.515 & $0.011 *$ & 0.921 & 0.864 & 0.981 \\
\hline Diuretics & 0.64 & 0.379 & 2.867 & 0.090 & 1.900 & 0.904 & 3.994 \\
\hline P2Y 12 receptor inhibitors & 0.68 & 0.308 & 4.820 & $0.028 *$ & 1.965 & 1.075 & 3.592 \\
\hline Constant & 1.24 & $\mathrm{I} .478$ & 0.703 & 0.402 & 3.453 & & \\
\hline
\end{tabular}

Note: $* p<0.05$.

Abbreviations: HAPR, high on-aspirin platelet reactivity; Hct, hematocrit; OR, odds ratio; PLT, platelet count; SE, standard error; SUA, serum uric acid.

variables such as age, gender, type 2 diabetes mellitus, current smoking and serum lipids were considered. ${ }^{25-27}$ Variables listed in Table 1 with a $P$-value $<0.10$ were also selected, including eGFR, SUA, platelet count, hemoglobin, hematocrit, concomitant $\mathrm{P} 2 \mathrm{Y} 12$ receptor inhibitors and diuretics use. Table 2 lists the variables included in the equation at the last step, and all $P$-values have been adjusted for age and gender. It was revealed that SUA (OR: $1.004,95 \% \mathrm{CI}$ : $1.000-1.007, P=0.048)$, platelet count (OR: $0.994,95 \%$ CI: $0.989-1.000, P=0.045$ ), hematocrit (OR: $0.921,95 \%$ CI: $0.864-0.981, P=0.011)$ and concomitant $\mathrm{P} 2 \mathrm{Y} 12$ receptor inhibitors use (OR: 1.965, 95\% CI: $1.075-3.592, P=0.028$ ) were independently correlated with HAPR.

Spearman's correlation analysis was performed to evaluate the correlation between LTA-AA and these variables with a $P$-value $<0.10$. As shown in Figure 2, hemoglobin ( $r=-0.209, P<0.001)$, hematocrit $(r=-0.234, P<0.001)$ and eGFR ( $r=-0.132, P=0.031)$ were negatively associated with LTA-AA.

\section{Discussion}

Advanced age is associated with higher risk of thrombotic complications, with increased mortality and worse prognosis, ${ }^{28,29}$ which might be partly due to the increasing occurrence of HPR in this population. ${ }^{18,30}$ In our study, the percentage of senile patients gradually increased from LAPR to HAPR group. The relatively higher proportion of senile patients in HAPR group might be due to declined absorption of aspirin, drug-drug interactions and age-related hemodynamic changes in the elderly. As the link between HAPR with
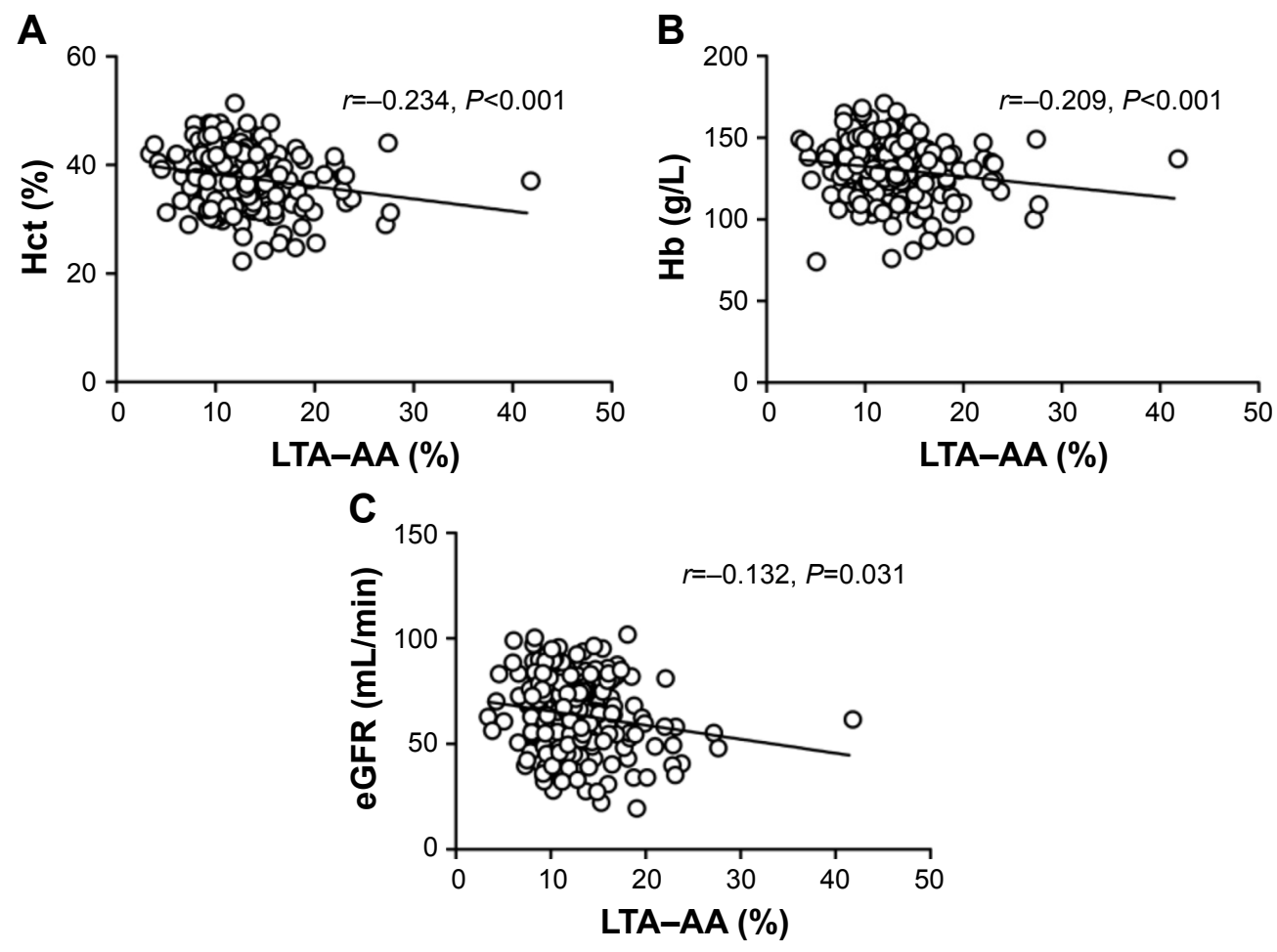

Figure 2 Spearman's correlation analysis.

Notes: (A) Hematocrit $(r=-0.234, P<0.001)$ was negatively associated with LTA-AA. (B) Hemoglobin $(r=-0.209, P<0.00$ I $)$ was inversely correlated with LTA-AA. (C) eGFR $(r=-0.132, P=0.031)$ was inversely correlated with LTA-AA.

Abbreviations: eGFR, estimated glomerular filtration rate; Hb, hemoglobin; Hct, hematocrit; LTA-AA, light transmission assay-arachidonic acid. 
cardiovascular events has been substantially illustrated, it is crucial to reveal predictors of HAPR and uncover potential mechanisms, in order to identify at-risk patients and to optimize antiplatelet therapy, especially in the elderly.

In our study, HAPR patients tended to have reduced renal function, and Spearman's correlation analysis confirmed an inverse association between LTA-AA and eGFR. Similarly, Blann et al observed a weak relationship between lower eGFR and HAPR, regardless of the method for platelet reactivity evaluation. What is more, eGFR even showed an inverse correlation with platelet expression of CD62P. ${ }^{31}$ Aksu et al observed higher incidence of HAPR in patients with severe chronic kidney disease (CKD), but not in mild or moderate CKD. HAPR might contribute to the increasing risk of cardiovascular complications in patients with declined renal function. ${ }^{32}$ However, Tello-Montoliu et al failed to track the link between antiplatelet response with renal dysfunction in non-diabetic patients with CAD. ${ }^{33}$ Our study excluded patients with severe CKD, thus minimizing the impact.

In our study, higher SUA level, as well as higher incidence of hyperuricemia was observed in HAPR patients. Previous studies reported the increasing risk of recurrent gout attacks in patients on low-dose aspirin. ${ }^{34}$ However, the link between SUA with aspirin response in the elderly has not been reported before. In the Nardin et al study, SUA level increased in patients receiving aspirin in combination with ticagrelor, but not in those with clopidogrel. However, their work failed to confirm effects of SUA on residual platelet reactivity. ${ }^{35}$ Similarly, Barbieri et al did not observe an association between SUA and HAPR. ${ }^{36}$ However, a weak correlation between HAPR with SUA was shown in our enrolled patients, and higher SUA level might potentially indicate the relatively poor response to aspirin. A possible explanation was that elevation of uric acid promotes the formation of a pro-inflammatory and prothrombotic state, with an increase in non-platelet TXA2 synthesis, thus leading to insufficient platelet inhibition. ${ }^{37}$

Elderly HAPR patients had lower platelet count, when compared with non-HAPR patients. Likewise, platelet count was weakly correlated with HAPR in our study. It was conventionally thought that platelet counts might influence the results of platelet aggregation studies. However, recent studies revealed that LTA is not sensitive to platelet count changes, at least within a broad range of $150-600 \times 10^{9} / \mathrm{L} .{ }^{38,39}$ In ADAPT-DES (Assessment of Dual AntiPlatelet Therapy with Drug-Eluting Stents) study, lower platelet count was associated with higher $\mathrm{P} 2 \mathrm{Y} 12$ reaction units (PRU), but after multivariate adjustment, higher platelet count was independently associated with increased thrombosis risks. ${ }^{40}$ It was also reported that aspirin might promote platelet apoptosis via several mechanisms, including the activation of p38 map kinase (MAPK), protein kinase C/B (PKC/PKB) and caspase-3, inhibition of proteasome function, triggering phosphatidylserine externalization and so forth. ${ }^{41-43}$

An inverse association of LTA-AA with hemoglobin and hematocrit was observed in our study. Previous studies reported the negative association between hematocrit or hemoglobin with VerifyNow assays or multiple electronic assay results, but not with LTA. In Voisin et al study, while PRU significantly declined, hematocrit and hemoglobin increased, but the percentage of platelet inhibition did not vary. ${ }^{44}$ In Pendyala et al study, lower baseline hematocrit was linked with HPR evaluated by the VerifyNow assay. ${ }^{45}$ Kim et al confirmed an inverse correlation between VerifyNow assay results with hematocrit ( $r=-0.409, P=0.001$ ), and after adjustment, anemia was an independent risk factor for HPR. ${ }^{46}$ A possible explanation is that hematocrit changes might influence calcium concentration in citrated blood used for PFTs. Thus, Kakouros et al developed a formula for PRU adjustment according to hematocrit. ${ }^{47,48}$

Concomitant drugs might influence antiplatelet effects as well. Our study revealed the proportion of diuretic use was higher in HAPR patients when compared with non-HAPR patients. However, it is inadequate to establish a causal relationship, and the potential mechanisms warrant further study. Further, concomitant P2Y12 receptor inhibitor use was correlated with HAPR, possibly due to the tendency of more P2Y12 receptor inhibitor use in patients with poor aspirin response to obtain sufficient platelet inhibition. Furthermore, previous studies reported possible effects of PPIs, calcium channel blockers (CCBs) or statins on antiplatelet responses, while controversies existed. In Liu et al, esomeprazole or rabeprazole use did not affect antiplatelet response after initiation of DAPT for 30 days. ${ }^{49}$ ADAPT-DES study demonstrated the interaction between combined PPIs use with clopidogrel (OR: 1.38, 95\% CI: 1.25-1.52, P<0.001). ${ }^{50}$ Tsukahara et al reported that CCB use was associated with higher incidence of HPR (OR: 1.93, 95\% CI: 1.18-3.18, $P<0.05) .{ }^{51}$ In Gremmel et al, co-administration with CCB was an independent risk factor of HPR, based on both LTA assay and VerifyNow assay. ${ }^{52}$ In terms of statins effects, it was reported that switching to a non-CYP3A4 metabolized statin might minimize drug-drug interactions and improve antiplatelet responses. ${ }^{53}$ However, Malmstrom et al revealed that lipid-lowering treatments did not exert additional inhibitory effects on platelets in CAD patients with impaired glucose tolerance. ${ }^{54}$

The present studies have some advantages. First, the mean age of our enrolled patients were 77.2 years, higher 
than most previous studies, which focused on HAPR. As older age has been recognized as a crucial risk factor for HAPR, investigating predictors of HAPR in this population might promote the optimization of antiplatelet therapy and uncover potential mechanisms. Further, ADP-induced platelet aggregation was used to exclude patients with poor response to thienopyridines, thus minimizing interference factors. Furthermore, all LTA assays in this study were performed by skilled technicians in our clinical laboratory, and results were normalized according to platelet counts.

Nevertheless, there are a few limitations. Mainly, this is a single-center study and other PFTs such as VerifyNow aspirin, multiple electrode platelet aggregometry or PFA-100 were not performed simultaneously.

In conclusion, elderly HAPR patients tended to have reduced renal function, higher SUA level as well as lower platelet count, hemoglobin and hematocrit. Platelet count, SUA, hematocrit and concomitant P2Y12 receptor inhibitors use were independently correlated with HAPR. These parameters might provide novel therapeutic targets for optimizing antiplatelet therapy.

\section{Acknowledgement}

This study was funded by the International Science \& Technology Corporation Project of China [2013DFA30860] and the National Key Research \& Development Program of China [2016YFC1301304]. The sponsors had no role in the study design, data collection, interpretation, or fulfillment of this manuscript.

\section{Disclosure}

The authors report no conflicts of interest in this work.

\section{References}

1. Engelmann B, Massberg S. Thrombosis as an intravascular effector of innate immunity. Nat Rev Immunol. 2013;13(1):34-45.

2. Knowles RB, Lawrence MJ, Ferreira PM, et al. Platelet reactivity influences clot structure as assessed by fractal analysis of viscoelastic properties. Platelets. 2017:1-9.

3. Li J, Tan M, Xiang Q, Zhou Z, Yan H. Thrombin-activated plateletderived exosomes regulate endothelial cell expression of ICAM-1 via microRNA-223 during the thrombosis-inflammation response. Thromb Res. 2017;154:96-105.

4. Broos K, Trekels M, Jose RA, et al. Identification of a small molecule that modulates platelet glycoprotein Ib-von Willebrand factor interaction. J Biol Chem. 2012;287(12):9461-9472.

5. Viles-Gonzalez JF, Fuster V, Badimon JJ. Atherothrombosis: a widespread disease with unpredictable and life-threatening consequences. Eur Heart J. 2004;25(14):1197-1207.

6. Fontana P, Zufferey A, Daali Y, Reny JL. Antiplatelet therapy: targeting the TxA2 pathway. J Cardiovasc Transl Res. 2014;7(1):29-38.

7. Floyd CN, Ferro A. Mechanisms of aspirin resistance. Pharmacol Ther. 2014;141(1):69-78.

8. Coccheri S. Antiplatelet therapy: controversial aspects. Thromb Res. 2012; 129(3):225-229.
9. Breet NJ, van Werkum JW, Bouman HJ, et al. High on-treatment platelet reactivity to both aspirin and clopidogrel is associated with the highest risk of adverse events following percutaneous coronary intervention. Heart. 2011;97(12):983-990.

10. Mayer K, Bernlochner I, Braun S, et al. Aspirin treatment and outcomes after percutaneous coronary intervention: results of the ISAR-ASPI registry. J Am Coll Cardiol. 2014;64(9):863-871.

11. Kuzniatsova N, Shantsila E, Blann A, Lip GYH. A contemporary viewpoint on 'aspirin resistance'. Ann Med. 2012;44(8):773-783.

12. Han YL. Aspirin resistance in coronary heart disease: current understandings and strategies. $J$ Transl Int Med. 2016;4(1):7-10.

13. Krasopoulos G, Brister SJ, Beattie WS, Buchanan MR. Aspirin "resistance" and risk of cardiovascular morbidity: systematic review and meta-analysis. BMJ. 2008;336(7637):195-198.

14. Wisman PP, Roest M, Asselbergs FW, et al. Platelet-reactivity tests identify patients at risk of secondary cardiovascular events: a systematic review and meta-analysis. J Thromb Haemost. 2014;12(5):736-747.

15. Gorog DA, Fuster V. Platelet function tests in clinical cardiology: unfulfilled expectations. J Am Coll Cardiol. 2013;61(21):2115-2129.

16. Gorog DA, Otsui K, Inoue N. Usefulness of platelet function tests to predict bleeding with antithrombotic medications. Cardiol Rev. 2015; 23(6):323-327.

17. Janssen PW, ten Berg JM. Platelet function testing and tailored antiplatelet therapy. J Cardiovasc Transl Res. 2013;6(3):316-328.

18. Verdoia M, Pergolini P, Rolla R, et al. Advanced age and high-residual platelet reactivity in patients receiving dual antiplatelet therapy with clopidogrel or ticagrelor. J Thromb Haemost. 2016;14(1):57-64.

19. Breet NJ, van Donkersgoed HE, van Werkum JW, et al. Is platelet inhibition due to thienopyridines increased in elderly patients, in patients with previous stroke and patients with low body weight as a possible explanation of an increased bleeding risk? Nether Heart J. 2011;19(6): 279-284.

20. Amsallem M, Manzo-Silberman S, Dillinger JG, et al. Predictors of high on-aspirin platelet reactivity in high-risk vascular patients treated with single or dual antiplatelet therapy. Am J Cardiol. 2015;115(9): 1305-1310.

21. Gum PA, Kottke-Marchant K, Welsh PA, White J, Topol EJ. A prospective, blinded determination of the natural history of aspirin resistance among stable patients with cardiovascular disease. J Am Coll Cardiol. 2003;41(6):961-965.

22. Jeong YH, Lee SW, Choi BR, et al. Randomized comparison of adjunctive cilostazol versus high maintenance dose clopidogrel in patients with high post-treatment platelet reactivity: results of the ACCELRESISTANCE (adjunctive cilostazol versus high maintenance dose clopidogrel in patients with clopidogrel resistance) randomized study. J Am Coll Cardiol. 2009;53(13):1101-1109.

23. Lemesle G, Landel JB, Bauters A, et al. Poor agreement between light transmission aggregometry, Verify Now P2Y(1)(2) and vasodilatatorstimulated phosphoprotein for clopidogrel low-response assessment: a potential explanation of negative results of recent randomized trials. Platelets. 2014;25(7):499-505.

24. Choi H, Kim HC, Song BM, et al. Serum uric acid concentration and metabolic syndrome among elderly Koreans: The Korean Urban Rural Elderly (KURE) study. Archiv Gerontol Geriatr. 2016;64:51-58.

25. Price MJ, Nayak KR, Barker CM, Kandzari DE, Teirstein PS. Predictors of heightened platelet reactivity despite dual-antiplatelet therapy in patients undergoing percutaneous coronary intervention. Ame J Cardiol. 2009;103(10):1339-1343.

26. Kaplon-Cieslicka A, Rosiak M, Postula M, et al. Predictors of high platelet reactivity during aspirin treatment in patients with type 2 diabetes. Kardiol Pol. 2013;71(9):893-902.

27. Winter MP, Kozinski M, Kubica J, Aradi D, Siller-Matula JM. Personalized antiplatelet therapy with P2Y12 receptor inhibitors: benefits and pitfalls. Postepy Kardiol Interwencyjnej. 2015;11(4):259-280.

28. Andreotti F, Rocca B, Husted S, et al. Antithrombotic therapy in the elderly: expert position paper of the European Society of Cardiology Working Group on Thrombosis. Eur Heart J. 2015;36(46): $3238-3249$. 
29. Hamon M, Lemesle G, Tricot O, et al. Incidence, source, determinants, and prognostic impact of major bleeding in outpatients with stable coronary artery disease. J Am Coll Cardiol. 2014;64(14):1430-1436.

30. Silvain J, Cayla G, Hulot JS, et al. High on-thienopyridine platelet reactivity in elderly coronary patients: the SENIOR-PLATELET study. Eur Heart J. 2012;33(10):1241-1249.

31. Blann AD, Kuzniatsova N, Velu S, Lip GY. Renal function and aspirin resistance in patients with coronary artery disease. Thromb Res. 2012; 130(3):e103-e106.

32. Aksu HU, Oner E, Erturk M, et al. Aspirin resistance in patients with impaired renal functions. Kardiol Pol. 2014;72(4):331-338.

33. Tello-Montoliu A, Ferreiro JL, Kodali MK, et al. Impact of renal function on clopidogrel-induced antiplatelet effects in coronary artery disease patients without diabetes mellitus. J Thromb Thrombolysis. 2013; 36(1):14-17.

34. Zhang Y, Neogi T, Chen C, Chaisson C, Hunter DJ, Choi H. Low-dose aspirin use and recurrent gout attacks. Ann Rheum Dis. 2014;73(2): 385-390.

35. Nardin M, Verdoia M, Pergolini P, et al. Serum uric acid levels during dual antiplatelet therapy with ticagrelor or clopidogrel: results from a singlecentre study. Nutr Metab Cardiovasc Dis. 2016;26(7):567-574.

36. Barbieri L, Verdoia M, Pergolini P, et al. Uric acid and high-residual platelet reactivity in patients treated with clopidogrel or ticagrelor. Nutr Metab Cardiovasc Dis. 2016;26(4):352-358.

37. Zapolski T, Wacinski P, Kondracki B, Rychta E, Buraczynska MJ, Wysokinski A. Uric acid as a link between renal dysfunction and both pro-inflammatory and prothrombotic state in patients with metabolic syndrome and coronary artery disease. Kardiol Pol. 2011;69(4): 319-326.

38. Podda G, Femia EA, Cattaneo M. Current and emerging approaches for evaluating platelet disorders. Int J Lab Hematol. 2016;38(Suppl 1): 50-58.

39. Femia EA, Scavone M, Lecchi A, Cattaneo M. Effect of platelet count on platelet aggregation measured with impedance aggregometry (multiplate analyzer) and with light transmission aggregometry. J Thromb Haemost. 2013;11(12):2193-2196.

40. Giustino G, Kirtane AJ, Genereux P, et al. Relation between platelet count and platelet reactivity to thrombotic and bleeding risk: from the assessment of dual antiplatelet therapy with drug-eluting stents study. Am J Cardiol. 2016;117(11):1703-1713.

41. Zhao L, Zhang W, Chen M, Zhang J, Zhang M, Dai K. Aspirin Induces platelet apoptosis. Platelets. 2013;24(8):637-642.

42. Thushara RM, Hemshekhar M, Kemparaju K, Rangappa KS, Devaraja S, Girish KS. Therapeutic drug-induced platelet apoptosis: an overlooked issue in pharmacotoxicology. Archiv Toxicol. 2014;88(2):185-198.
43. Nayak MK, Dash A, Singh N, Dash D. Aspirin delimits platelet life span by proteasomal inhibition. PloS One. 2014;9(8):e105049.

44. Voisin S, Bongard V, Tidjane MA, Lhermusier T, Carrie D, Sie P. Are P2Y12 reaction unit (PRU) and \% inhibition index equivalent for the expression of P2Y12 inhibition by the VerifyNow assay? Role of haematocrit and haemoglobin levels. Thromb Haemost. 2011;106(2): 227-229.

45. Pendyala LK, Loh JP, Lhermusier T, et al. Does baseline hematocrit influence the assays of on-treatment platelet reactivity to clopidogrel? Am Heart J. 2014;168(4):545-551.

46. Kim YG, Suh JW, Park JJ, et al. Different influences of hematocrit on the results of two Point-Of-Care platelet function tests, the VerifyNow assay and multiple electrode platelet aggregometry. PloS One. 2014; 9(11):e114053.

47. Janssen PW, Bergmeijer TO, Godschalk TC, et al. The effect of correcting VerifyNow $\mathrm{P} 2 \mathrm{Y} 12$ assay results for hematocrit in patients undergoing percutaneous coronary interventions. J Thromb Haemost. 2017;15:1-6.

48. Kakouros N, Kickler TS, Laws KM, Rade JJ. Hematocrit alters VerifyNow P2Y12 assay results independently of intrinsic platelet reactivity and clopidogrel responsiveness. J Thromb Haemost. 2013; 11:1814-1822.

49. Liu LP, Wang Y, Si R, Yuan M, Cheng K, Guo WY. Esomeprazole and rabeprazole did not reduce antiplatelet effects of aspirin/clopidogrel dual therapy in patients undergoing percutaneous coronary intervention: a prospective, randomized, case-control study. Expert Opin Pharmacother. 2016;17(1):7-16.

50. Weisz G, Smilowitz NR, Kirtane AJ, et al. Proton pump inhibitors, platelet reactivity, and cardiovascular outcomes after drug-eluting stents in clopidogrel-treated patients: the ADAPT-DES Study. Circ Cardiovasc Interv. 2015;8(10):e001952.

51. Tsukahara K, Kimura K, Morita S, et al. Impact of concomitant use of proton-pump inhibitors and thienopyridine derivatives on the antiplatelet effects. J Cardiol. 2011;57(3):275-282.

52. Gremmel T, Steiner S, Seidinger D, Koppensteiner R, Panzer S, Kopp CW. Calcium-channel blockers decrease clopidogrel-mediated platelet inhibition. Heart. 2010;96(3):186-189.

53. Leoncini M, Toso A, Maioli M, Bellandi F. [Statin and clopidogrel pharmacological interaction]. Giornale Italiano Di cardiologia. 2013; 14(9):574-584. Italian.

54. Malmstrom RE, Settergren M, Bohm F, Pernow J, Hjemdahl P. No effect of lipid lowering on platelet activity in patients with coronary artery disease and type 2 diabetes or impaired glucose tolerance. Thromb Haemost. 2009;101(1):157-164. 


\section{Supplementary materials}

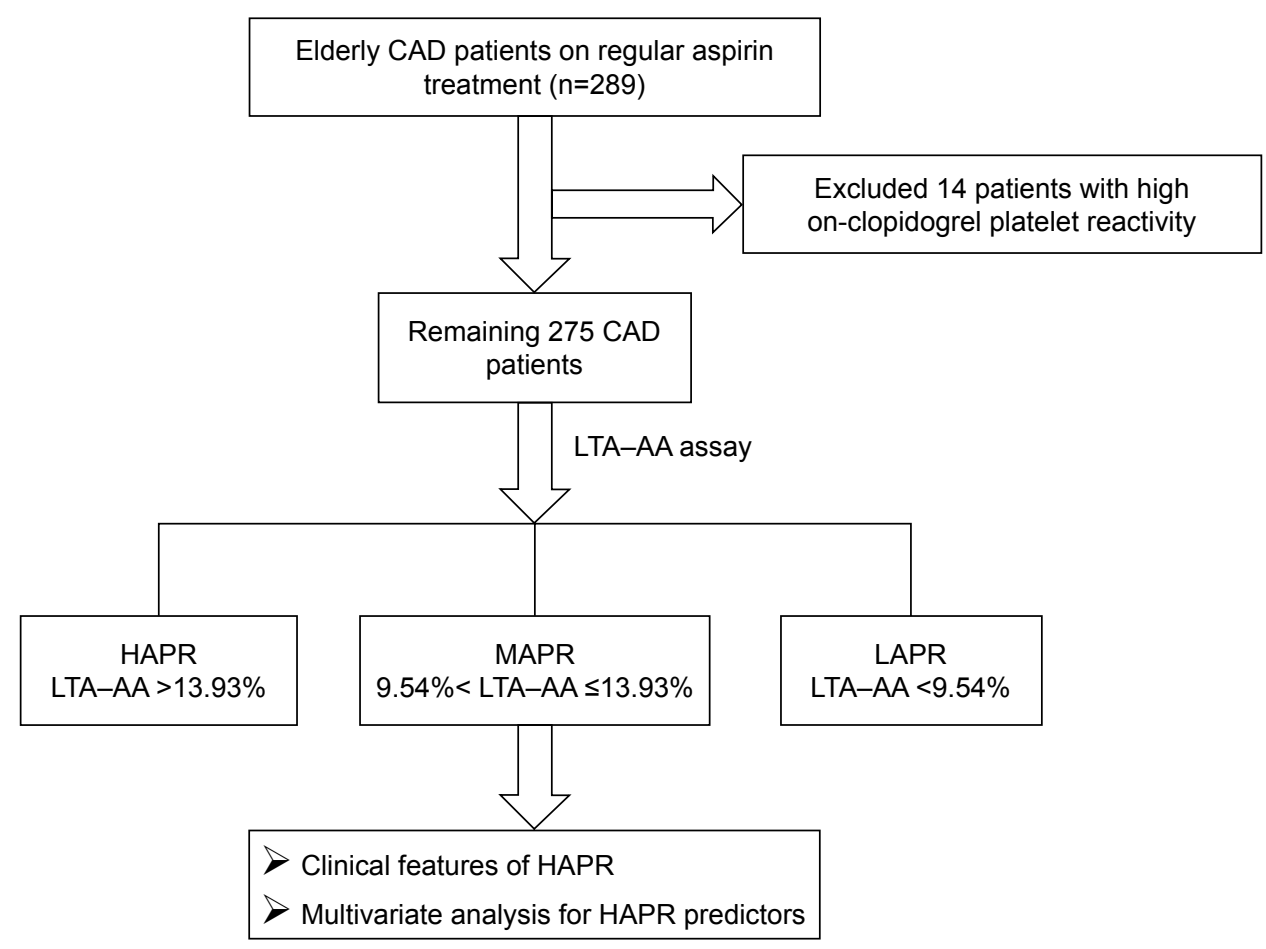

Figure SI Flowchart of the study.

Abbreviations: CAD, coronary artery disease; HAPR, high on-aspirin platelet reactivity; LAPR, low on-aspirin platelet reactivity; LTA-AA, light transmission assayarachidonic acid; MAPR, moderate on-aspirin platelet reactivity.

Table SI Predictive value for HAPR (ROC curve)

\begin{tabular}{lllll}
\hline Variables & AUC & P-value & 95\% Lower & 95\% Upper \\
\hline SUA & 0.625 & $0.002 * *$ & 0.545 & 0.706 \\
PLT & 0.580 & 0.052 & 0.499 & 0.662 \\
eGFR & 0.593 & $0.024^{*}$ & 0.511 & 0.676 \\
Hb & 0.578 & 0.060 & 0.500 & 0.656 \\
Hct & 0.595 & $0.021^{*}$ & 0.518 & 0.673 \\
Combination (SUA + PLT + eGFR + Hb + Hct) & 0.661 & $<0.001 * * *$ & 0.581 & 0.740 \\
\hline
\end{tabular}

Notes: $* P<0.05, * * P<0.01, * * * P<0.001$.

Abbreviations: AUC, area under the curve; eGFR, estimated glomerular filtration rate; HAPR, high on-aspirin platelet reactivity; Hb, hemoglobin; Hct, hematocrit; PLT, platelet count; ROC, receiver operator characteristic; SUA, serum uric acid.

Clinical Interventions in Aging

Dovepress

\section{Publish your work in this journal}

Clinical Interventions in Aging is an international, peer-reviewed journal focusing on evidence-based reports on the value or lack thereof of treatments intended to prevent or delay the onset of maladaptive correlates of aging in human beings. This journal is indexed on PubMed Central, MedLine,
CAS, Scopus and the Elsevier Bibliographic databases. The manuscript management system is completely online and includes a very quick and fair peer-review system, which is all easy to use. Visit http://www.dovepress. com/testimonials.php to read real quotes from published authors. 